\title{
A Novel Algorithm for the Calibration of Inertial/Magnetic Sensors: Application to a Body Sensor Network
}

\author{
Daniel Rüschen ${ }^{1 *}$, Berno J.E. Misgeld ${ }^{1}$, Saim Kim² and Steffen Leonhardt ${ }^{1}$ \\ 'Philips Chair for Medical Information Technology, RWTH Aachen University, Aachen, Germany \\ ${ }^{2}$ MELAG Medizintechnik oHG, Berlin, Germany
}

\section{BIOGRAPHICAL NOTES}

Daniel Rüschen, M.Sc., (born in 1986) received the B.Sc. and M.Sc. degrees from RWTH Aachen University, Aachen, Germany in 2010 and 2013, respectively. He is currently a scientific employee at the Philips Chair for Medical Information Technology, Helmholtz-Institute for Biomedical Engineering at RWTH Aachen University. His research interests include control of ventricular assist devices, rehabilitation robotics, and human body motion tracking using inertial/magnetic sensors.

RDr.-Ing. Berno J.E. Misgeld, M.Sc., (IFAC,IEE,VDA), born 1979, received the Dipl.-Ing. $(\mathrm{FH})$ in Electrical and Automation Engineering from University of Applied Sciences, Aachen, Germany, and the M.Sc. degree from Coventry University, Coventry, U.K., in 2003, respectively. In 2007 he received the Dr.-Ing. degree in Biomedical and Control Engineering from Ruhr-University Bochum, Bochum, Germany. From 2006 to 2011 he was a research and development engineer for guidance and flight control systems at Diehl-BGT-Defence, Ueberlingen, Germany. Since 2011 he is a Senior Scientific Engineer in Biomechatronical Systems and Control and Rehabilitation Robotics at the Chair of Medical Information Technology at RWTH Aachen University, Aachen, Germany. His research interests include feedback control and filtering with application to biomedical systems, robotics and medicine.

Dipl.-Ing. Saim Kim, (was born in 1980. He received the Dipl.-Ing. degree in electrical engineering with a specialization in electrical and electronic engineering from RWTH Aachen University, Aachen, in 2006. His research interests included Body Sensor Networks, personal health care systems and Bioimpedance Spectroscopy while working at the Philips Chair of Medical Information Technology, RWTH Aachen University. He is currently team leader of hardware development at MELAG Medizintechnik oHG and member of the VDE and IEEE.

Univ.-Prof. Dr.-Ing. Dr. med. Steffen Leonhardt, was born in Frankfurt, Germany, in 1961. He received the M.S. degree in computer engineering from SUNY, Buffalo, NY, USA, the Dipl.-Ing. In electrical engineering and the Dr.-Ing. degree in control engineering both from the Technische Universität Darmstadt, Darmstadt, Germany, and the M.D. degree in medicine from J. W. Goethe University, Frankfurt, Germany. He has 5 years of R\&D management experience in medical engineering industry and was appointed a Full Professor and the Head of the Philips endowed Chair of Medical Information Technology, RWTH Aachen University, Aachen, Germany, in 2003. His research interests include physiological measurement techniques, personal health care systems, and feedback control systems in medicine.

\section{ABSTRACT}


It can be used to estimate human posture in a dynamic environment with multiple applications ranging from artifact or disturbance detection to rehabilitation robotics. The development and test of inertial measurement units requires calibration and a proper reference that can be provided by a motion tracking system. We present a novel approach for the fast calibration of inertial/magnetic sensors and the spatial synchronization with a reference system. The calibration procedure is tested using a Body Sensor Network-based inertial measurement unit and a Vicon tracking system to observe body limb orientation during normal gait on a treadmill. The proposed algorithm reduces the errors incorporated by misalignment of the reference frames and decalibration in the utilized experimental setup by $8.8^{\circ}$.

\section{Introduction}

Accurate orientation information of human body segments is vital for applications ranging from physical rehabilitation and monitoring over virtual reality to advanced sports. Due to the enduring improvement of micro-electro-mechanical systems (MEMS), inertial/magnetic sensor based systems achieved a degree of technical maturity that provide reliable data at low-cost [1]. By using sophisticated data fusion methods, these systems can be long-term accurate without losing their short-term precision [2]. The integration of these sensors in wireless body sensor networks (BSNs) enables a broad range of new applications. We address the estimation of human motion kinematics with multiple inertial measurement units (IMUs) in this paper. The Integrated Posture and Activity NEtwork by Medit Aachen (IPANEMA) is used as a framework for multiple magnetic, angular rate and gravity (MARG) sensor nodes. The performance of these nodes is evaluated with a motion tracking system.

One of the challenges associated with this experimental setup is the spatial synchronization of the different reference and body frames of the IMU and the motion tracking system. The reference frame of the IMU is the so called navigation frame, its coordinate axes are earth-fixed and point to the magnetic north, east and down parallel to the gravitational acceleration vector. The IMU body frame is determined by the alignment of the sensitive axis of the particular sensors. Whereas the reference frame of the motion tracking system as well as the body frame of the markers attached to the IMU can be arbitrarily chosen during the initial setup. We propose a novel method based on convex optimization that enables the estimation of the transformations between the different coordinate frames and the calibration of an IMU at one go.

This paper is organized as follows. Section 2 briefly describes the IPANEMA BSN and the utilized hardware. Section 3 introduces the underlying system and sensor model, followed by a complete overview of the proposed calibration procedure in section 4. Finally, the results are presented in section 5 and conclusions are drawn in section 6 .

\section{Body Sensor Network and Inertial/Magnetic Sensor Nodes}

The IPANEMA BSN provides a wearable and flexible platform that enables mobile measurements in a wide range of medical and health-oriented scenarios [3]. It utilizes a star network topology where multiple slave nodes communicate with a master node in the $433 \mathrm{MHz}$ industrial, scientific and medical (ISM) band using a time division multiple access (TDMA) scheme. The core of each BSN node is a microcontroller (MSP430F1611, Texas Instruments Inc., USA) that operates the main functional units such as the wireless transceiver (CC1101, Texas Instruments Inc., USA). Additionally, it provides extension ports for custom functional units including sensors and actuators. We use such an extension board equipped with triaxial inertial and magnetic sensors for real-time strapdown orientation estimation.

Figure 1 shows a functional block diagram of the sensor node. The $I^{2} \mathrm{C}$ (Inter-Integrated Circuit) bus with the microcontroller as master and the sensors as slaves is used for on board communication. The gyroscope (ITG-3200, InvenSense Inc., USA) and the acceleration sensor (ADXL312, Analog Devices Inc., USA) are based on MEMS technology, the magnetic field sensor (HMC5883L, Honeywell International Inc., USA) uses magneto-resistive elements to measure the magnetic field strength. MEMS inertial sensors are small and provide lowcost and low-energy measurements of the gravitational acceleration and the angular velocity of the device. However, especially low-cost sensors still lack of bias-stability and have a comparatively high spectral noise density. Hence, a sophisticated data 


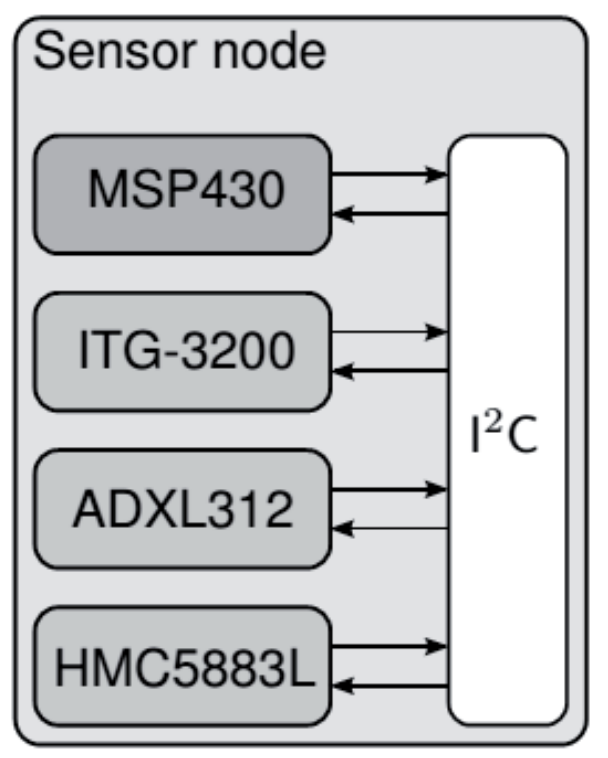

Fig. 1: Functional block diagram of the proposed BSN node for orientation estimation.

fusion method such as the extended Kalman filter is required for robust orientation estimation.

\section{Modeling and Estimation of Spatial Orientation}

The orientation of a rigid body in space is described by the transformation from an arbitrarily attached coordinate frame to a reference frame. The former is called the body frame (denoted by the superscript b), the latter is usually named the navigation frame (labeled with $\mathrm{n}$ ). A common way to describe the rotation between these frames is a direction cosine matrix (DCM) $\mathbf{C}_{b}^{n}$. It is defined as an orthogonal $3 \times 3$ matrix with determinant 1 . A DCM can either be used to transform vectors described in the body frame to the navigation frame using:

$$
\mathbf{u}^{n}=\mathbf{C}_{b}^{n} \mathbf{u}^{b}
$$

or serve as a characterization of the orientation of the body frame itself. However, it is more efficient to use an orientation quaternion:

$\mathbf{q}=\left[\begin{array}{l}q_{1} \\ q_{2} \\ q_{3} \\ q_{4}\end{array}\right]=\left[\begin{array}{l}q_{1} \\ \mathbf{e}\end{array}\right] \in \mathbb{H}$

for this purpose, where the elements of $\mathbf{q}$ are sca- lars and $\mathbb{H}$ the set of all quaternions. $\mathrm{q}_{1}$ is defined as the scalar part and $\mathbf{e}$ as the imaginary part of the quaternion [4]. In order to use this as a representation of orientation, it is useful to define the quaternion norm and adjoint

$$
\begin{aligned}
& \mathbf{q}=\sqrt{q_{1}^{2}+q_{2}^{2}+q_{3}^{2}+q_{4}^{2}} \\
& \mathbf{q}^{+}=\left[\begin{array}{c}
q_{1} \\
-\mathrm{e}
\end{array}\right] .
\end{aligned}
$$

Only unit quaternions $(\mathbf{q}=1)$ represent valid orientations [5], they can be transformed in an equivalent DCM using

$$
\mathbf{C}(\mathbf{q})=\left(\mathbf{q}_{1}^{2}-\mathbf{e}^{2}\right) \mathbf{I}_{3}+2 \mathbf{e} \mathbf{e}^{\mathrm{T}}-2 q_{1}[\mathbf{e} \times]
$$

where: $\mathbf{I}_{3}$ is the $3 \times 3$ identity matrix and the operator $[\mathbf{e x}]$ is the skew-symmetric matrix of a vector product.

\subsection{Assistive Robots and Orthoses}

In order to find a useful system model, a relation between orientation and at least one of the available sensor outputs is needed. This relation is given by the vector differential equation:

$$
\dot{\mathbf{q}}=\frac{1}{2} \mathbf{q} \otimes\left[\begin{array}{l}
0 \\
\omega
\end{array}\right]
$$

where: $\otimes$ denotes quaternion multiplication and $\boldsymbol{\omega}$ is the angular velocity the rigid body. Formula 6 is a part of the following nonlinear state-space model from [6], which uses the three components of angular velocity and four elements of the orientation quaternion as states:

$$
\begin{aligned}
& {\left[\begin{array}{l}
\dot{\omega}_{1} \\
\dot{\omega}_{2} \\
\dot{\omega}_{3}
\end{array}\right]=\left[\begin{array}{l}
\dot{x}_{1} \\
\dot{x}_{2} \\
\dot{x}_{3}
\end{array}\right]=\frac{1}{\tau}\left[-\left[\begin{array}{l}
x_{1} \\
x_{2} \\
x_{3}
\end{array}\right]+\left[\begin{array}{l}
w_{1} \\
w_{2} \\
w_{3}
\end{array}\right]\right)} \\
& {\left[\begin{array}{l}
\dot{q}_{1} \\
\dot{q}_{2} \\
\dot{q}_{3} \\
\dot{q}_{4}
\end{array}\right]=\left[\begin{array}{c}
\dot{x}_{4} \\
\dot{x}_{5} \\
\dot{x}_{6} \\
\dot{x}_{7}
\end{array}\right]=\frac{1}{2}\left[\begin{array}{l}
x_{4} \\
x_{5} \\
x_{6} \\
x_{7}
\end{array}\right] \otimes\left[\begin{array}{l}
0 \\
x_{1} \\
x_{2} \\
x_{3}
\end{array}\right] .}
\end{aligned}
$$

The vector $\mathbf{w}$ is assumed to be white Gaussian noise representing unknown human movement. The parameter $\tau$ determines the bandwidth of the first order linear system modeling the transition from this movement to an angular velocity. The 
measurement model is based on the assumption that all states are directly measurable, only disturbed by additive noise:

$$
\mathbf{z}=\mathbf{x}+\mathbf{v}
$$

where: $\mathbf{v}=\left[\begin{array}{ll}\mathbf{V}_{\omega} & \mathbf{V}_{q}\end{array}\right]^{\mathrm{T}}$ is zero-mean white Gaussian measurement noise. The angular velocity can be measured with a gyroscope, the quaternion "measurement" is in fact the result of a one-step procedure called QUaternion ESTimator (QUEST).

\subsection{Sensor Model}

In addition to the system model, the triaxial MARG sensors with measured angular velocity $\tilde{\omega}$, acceleration $\tilde{\mathbf{a}}$, and magnetic field strength $\tilde{\mathbf{m}}$ are modeled by:

$$
\begin{aligned}
& \tilde{\omega}=\mathbf{K}_{\omega} \omega^{n}+\mathbf{b}_{\omega}+\mathbf{v}_{\omega} \\
& \tilde{\mathbf{a}}=\mathbf{K}_{a}\left[\mathbf{C}_{n}^{b}(\mathbf{q})\left(\mathbf{g}^{n}+\mathbf{a}^{n}\right)\right]+\mathbf{b}_{a}+\mathbf{v}_{a} \\
& \tilde{\mathbf{m}}=\mathbf{K}_{m} \mathbf{C}_{n}^{b}(\mathbf{q}) \mathbf{h}^{n}+\mathbf{b}_{m}+\mathbf{v}_{m} .
\end{aligned}
$$

The basic structure of the measurement equations is similar. The scale factors, cross-axis sensitivity, and misalignment are modeled by the matrices $\mathbf{K}_{\omega}, \mathbf{K}_{a}, \mathbf{K}_{m} \in \mathbb{R}^{3 \times 3}$. The vectors $\mathbf{b}_{\omega}, \mathbf{b}_{a}, \mathbf{b}_{m} \in \mathbb{R}^{3}$ contain biases and $\mathbf{v}_{\omega}, \mathbf{v}_{a}, \mathbf{v}_{m} \in \mathbb{R}^{3}$ are uncorrelated zero-mean white Gaussian measurement noise vectors [7],[8]. The measured acceleration is in fact the sum of the gravitational vector $\mathbf{g}^{n}=\left[\begin{array}{lll}0 & 0 & 9.81\end{array}\right]^{\mathrm{T}}$ and the actual acceleration $\mathbf{a}^{\mathrm{n}}$, rotated from the navigation in the body frame. The output of the magnetometer is the rotated and scaled earth's magnetic field strength vector $\mathbf{h}^{n}=h\left[\begin{array}{lll}\cos \alpha & 0 & \sin \alpha\end{array}\right]^{\mathrm{T}}$ with amplitude $\boldsymbol{h}$ and inclination angle $\boldsymbol{\alpha}$.

\subsection{Filter Design}

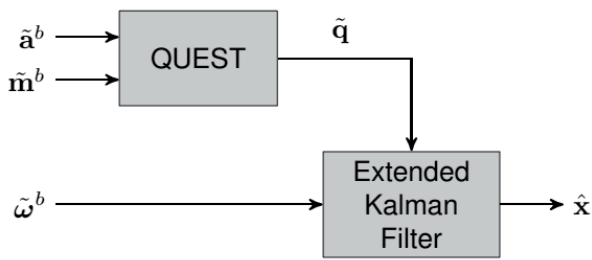

Fig. 2: Block diagram of the extended Kalman filter design with QUEST preprocessing.

The filter design proposed in [6] is depicted in Figure 2. It is different from the standard Extended Kalman Filter design in terms of the measurement vector. Instead of using the measured acceleration and magnetic field strength as input, the output of a QUEST is treated as a measurement. The QUEST algorithm determines an optimal orientation quaternion by solving Wahba's problem [9]:

$$
\underset{\mathbf{q}}{\operatorname{minimize}} \frac{1}{2} \sum_{i} a_{i}\left|\mathbf{b}_{i}-\mathbf{C}(\mathbf{q}) \mathbf{r}_{i}\right|^{2} .
$$

The vectors $\mathbf{b}_{\text {i }}$ contain the measurements of the acceleration and the magnetic field strength in the body frame, $\mathbf{r}_{\text {i }}$ are earth's gravitational acceleration and magnetic field, respectively. A detailed description of the QUEST algorithm can be found in [10].

The Extended Kalman Filter uses a first-order Taylor approximation of the nonlinear system model defined in eqn. (7) and the linear measurement model from eqn. (8). The standard equations of the time-varying Kalman Filter are implemented using these models.

\section{Calibration}

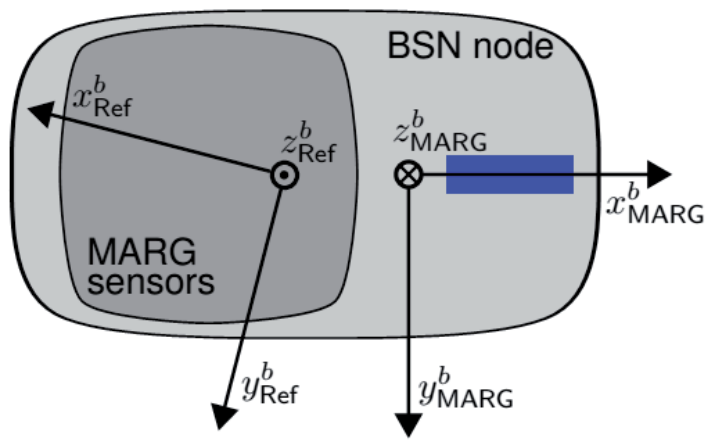

Fig. 3: BSN Node with mounted MARG sensors extension and different body coordinate frames.

The purpose of a calibration procedure is to determine the parameters of the sensor model introduced in eqns. (9). Additionally, the coordinate transformations between the motion tracking system and the MARG sensors body and reference frames have to be determined. The MARG sensor node uses the navigation frame as a reference, the orientation measured by the motion tracking system is related to an arbitrarily defined coordinate frame. Moreover, the body coordinate frames differ as well. Figure 3 illustrates the sensor position and these different coordinate frames. The DCM that 
rotates the reference body frame in the MARG sensors body frame is referred to as $\mathbf{C}^{\mathrm{b}}$. The transformation that rotates the tracking system reference frame in the navigation frame is denoted $\mathbf{C}^{\mathrm{n}}$.

The proposed calibration procedure consists of two different trials, which require continuously recording of MARG sensor and tracking data. In the first one, the sensor is at absolute rest yielding the mean values of the MARG sensors $\tilde{\omega}_{\text {stat }}, \tilde{\mathbf{a}}_{\text {stat }}, \tilde{\mathbf{m}}_{\text {stat }} \in \mathbb{R}^{3}$ and the motion capture signal. $\mathbf{q}_{\text {stat }} \in \mathbb{H}$ In the second trial, the BSN node is freely moved by hand inside the tracking volume yielding:

$$
\begin{aligned}
& \boldsymbol{\Omega}^{*}=\left[\begin{array}{llll}
\tilde{\omega}_{\mathrm{dyn}, 1} & \tilde{\omega}_{\mathrm{dyn}, 2} & \cdots & \tilde{\omega}_{\mathrm{dyn}, \mathrm{n}}
\end{array}\right] \\
& \mathbf{A}^{*}=\left[\begin{array}{llll}
\tilde{\mathbf{a}}_{\mathrm{dyn}, 1} & \tilde{\mathbf{a}}_{\mathrm{dyn}, 2} & \cdots & \tilde{\mathbf{a}}_{\mathrm{dyn}, \mathrm{n}}
\end{array}\right] \\
& \mathbf{M}^{*}=\left[\begin{array}{llll}
\tilde{\mathbf{m}}_{\mathrm{dyn}, 1} & \tilde{\mathbf{m}}_{\mathrm{dyn}, 2} & \cdots & \tilde{\mathbf{m}}_{\mathrm{dyn}, \mathrm{n}}
\end{array}\right] \\
& \mathbf{Q}=\left[\begin{array}{llll}
\mathbf{q}_{\mathrm{dyn}, 1} & \mathbf{q}_{\mathrm{dyn}, 2} & \cdots & \mathbf{q}_{\mathrm{dyn}, \mathrm{n}}
\end{array}\right]
\end{aligned}
$$

As earth's rotation is negligible compared to the gyroscope's sensitivity, its bias is directly obtained from the first trial

$$
\mathbf{b}_{\omega}=\tilde{\omega}_{\text {stat }} .
$$

The measured angular velocity from eqn. (11) can be corrected giving

$\boldsymbol{\Omega}=\left[\begin{array}{llll}\tilde{\omega}_{\mathrm{dyn}, 1}-\mathbf{b}_{\omega} & \tilde{\omega}_{\mathrm{dyn}, 2}-\mathbf{b}_{\omega} & \cdots & \tilde{\omega}_{\mathrm{dyn}, \mathrm{n}}-\mathbf{b}_{\omega}\end{array}\right](13)$

The true angular velocity is obtained from the tracking system data by approximating the derivatives of $\mathbf{q}$ dyn,i using

$\left[\begin{array}{c}0 \\ \omega_{\mathrm{dyn}, i}\end{array}\right] \approx 2 \frac{\Delta \mathbf{q}_{\mathrm{dyn}, i}}{\Delta t} \otimes \mathbf{q}_{\mathrm{dyn}, i}^{+}$

for $\mathrm{i}=1,2, \ldots, n$ combined to

$$
\boldsymbol{\Omega}_{\mathrm{ref}}=\left[\begin{array}{llll}
\omega_{\mathrm{dyn}, 1} & \omega_{\mathrm{dyn}, 2} & \cdots & \omega_{\mathrm{dyn}, \mathrm{n}}
\end{array}\right]
$$

Now we are able to determine the transformation of the measured angular velocity $\boldsymbol{\Omega}$ into the true angular velocity $\boldsymbol{\Omega}_{\text {ref }}$ by calculating the matrix $\mathrm{T} \in \mathbb{R}^{3 \times 3}$ that minimizes

$$
\square \mathbf{\Omega}_{\text {ref }}-\mathbf{T} \boldsymbol{\Omega} \square_{2}
$$

The matrix $\mathbf{T}$ can be splitted using the polar de- composition described in [11]:

$$
\mathbf{T}=\mathbf{C}^{b} \mathbf{K}_{\omega}
$$

yielding the DCM that rotates the reference body frame in the MARG sensors body frame $\mathbf{C}^{\mathrm{b}}$ and the calibration matrix $\mathbf{K}_{\omega}$. In order to obtain the magnetometer's and accelerometer's calibration parameters, earth's acceleration and magnetic field are rotated in the reference body frame by

$$
\begin{aligned}
& \mathbf{G}=\mathbf{Q} \otimes \mathbf{g}^{n} 1_{1 \times n} \otimes \mathbf{Q}^{+} \\
& \mathbf{H}=\mathbf{Q} \otimes \mathbf{h}^{n} 1_{1 \times n} \otimes \mathbf{Q}^{+}
\end{aligned}
$$

where the quaternion multiplication and adjoint are applied column wise. The measured acceleration and magnetic field vectors are also rotated in the body reference frame using

$$
\begin{aligned}
& \mathbf{A}=\mathbf{C}^{b} \mathbf{A}^{*} \\
& \mathbf{M}=\mathbf{C}^{b} \mathbf{M}^{*} .
\end{aligned}
$$

These matrices should ideally be equal to $G$ and $\mathrm{H}$, but due to noise and the approximation of the derivative in eqn. (14) this is generally not true. The calibration values for the accelerometer and magnetometer are estimated by solving the convex optimization problems

$$
\begin{aligned}
& \text { minimize } \sqsupset \mathbf{K}_{a} \mathbf{A}-\mathbf{b}_{a} 1_{1 \times n}-\mathbf{G} \square_{1} \\
& \mathbf{K}_{a}, \mathbf{b}_{a} \\
& \text { minimize } \mathbf{K}_{m} \mathbf{M}-\mathbf{b}_{\boldsymbol{m}} 1_{1 \times n}-\mathbf{H} \square_{1} \\
& \mathbf{K}_{m}, \mathbf{b}_{m}
\end{aligned}
$$

without constraints in which all columns of $\mathbf{A}$ that do not satisfy $1-\epsilon<\tilde{\mathbf{a}}_{\mathrm{dyn}, \mathrm{i}}<1+\epsilon$ with a sufficiently high $\epsilon$ are neglected.

\section{Results}

The performance of the proposed calibration procedure is validated with the experimental setup depicted in figure 4. A subject was equipped with a BSN nodes at hip, thigh and ankle in order to monitor the orientation of these limb segments. Measurements of human gait were conducted on a treadmill (Ergo Run Medical 8, Daum Electronic $\mathrm{GmbH}$, Germany). The absolute error was determined with a motion tracking system (Vicon Bonita with 6 cameras, Vicon, USA) sampling at a rate of 

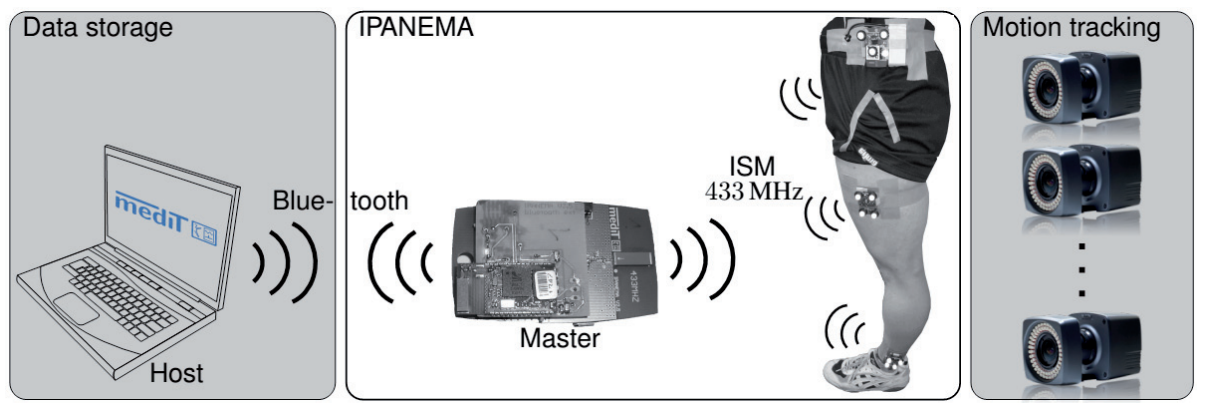

Fig. 4: Overview of the experimental setup.

$100 \mathrm{~Hz}$. The sampling frequency of the BSN-based IMUs were set to $75 \mathrm{~Hz}$.

Figure 5 shows Euler angles and estimation errors during normal gait at $3 \mathrm{~km} / \mathrm{h}$ on a treadmill. The solid lines are real data from the motion tracking system, the dashed and dotted lines are estimated by the Extended Kalman Filter from the uncalibrated and calibrated sensor signals, respectively. The reference frame of the motion tracking system manually set to match the navigation frame. This is done by a cross-shaped tool called "wand", which consists of two orthogonal levers equipped with water-levels. It has to be positioned in the tracking volume during system setup and defines the axes of the motion tracking reference frame. Figure 5 shows, that roll and pitch angles can be set quite accurately, but yaw angle that is defined by the magnetic north deviates strongly. This error is mostly corrected by the proposed calibration procedure.

\section{Conclusions}

We propose an algorithm, which determines the necessary transformations between an IMU and a motion tracking system that is capable of calculating the calibration values of the IMU simultaneously. The calibration method was validated in treadmill experiments with a BSN-based IMU. The orientation estimated from the calibrated signals is sufficient to allow a robust monitoring of the attitude of body segments. That enables the systematic investigation of the connection between human posture and other medically relevant data.

\section{References}

[1] Permutter, M., Robin, L. (2012). "High-performance, low cost inertial mems: A market in motion!". Position Location and Navigation Symposium, 225-229
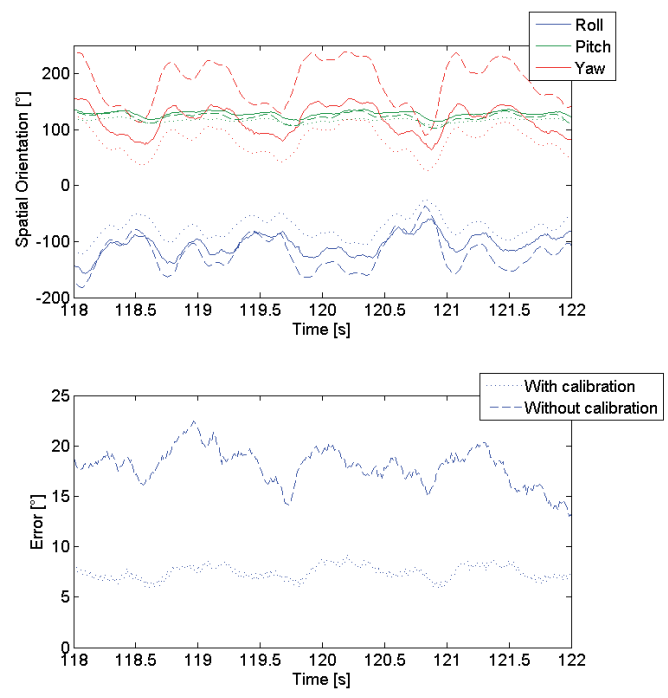

Fig. 5: Roll, pitch and yaw angles plus errors of normal human gait at $3 \mathrm{~km} / \mathrm{h}$. Solid lines are true values from the tracking system, dashed lines are without any calibration and dotted lines are calibration-corrected.

[2] Bruckner, H., Spindeldreier, C., Blume, H., Schoonderwaldt, E., Altenmuller, E. (2012).„Evaluation of inertial sensor fusion algorithms in grasping tasks using real input data: Comparison of computational costs and root mean square error". Int. Conf. on Wearable and Implantable Body Sensor Networks, 189-194

[3] Kim, S., Brendle, C., Lee, H.-Y., Walter, M., Gloeggler, S., Krueger, S., Leonhardt, S. (2013). „Evaluation of a 433 MHz band body sensor network for biomedical applications". Sensors, 13, 1, 898-917.

[4] Kuipers, J. (1999). "Quaternions and rotation sequences: A primer with applications to orbits, aerospace, and virtual reality". Princeton University Press, Princeton.

[5] Chou, J. (1992). "Quaternion kinematic and dynamic differential equations". IEEE Transactions on Robotics and Automation, 8, 1, 53-64.

[6] Yun, X., Bachmann, E. (2006). “Design, implementation, and 
experimental results of a quaternion-based kalman filter for human body motion tracking". IEEE Transactions on Robotics, 22, 6, 1216-1227.

[7] Ferraris, F., Grimaldi, U., Parvis, M. (1995). "Procedure for effortless in-field calibration of three-axis rate gyros and accelerometers". Sensors and Materials, 7, 5, 311-330.

[8] Gebre-Egziabher, D., Elkaim, G.H., Powell, J.D., Parkinson, B.W. (2001). "A non-linear, two-step estimation algorithm for calibrating solid-state strapdown magnetometers". Int. Conf. on Integrated Navigation Systems, 290-297.

[9] Wahba, G. (1965). "Problem 65-1: A least squares estimate of satellite attitude". SIAM Review, 7, 3, 409.

[10] Shuster, M.D., Oh, S.D. (1981). "Three-axis attitude determination from vector observations". Journal of Guidance Control and Dynamics, 4, 1, 70-77.

[11] Highham, N.J. (1986). "Computing the polar decomposition - with applications", SIAM Journal of Scientific and Statistical Computing, 7, 4, 1160-1174.

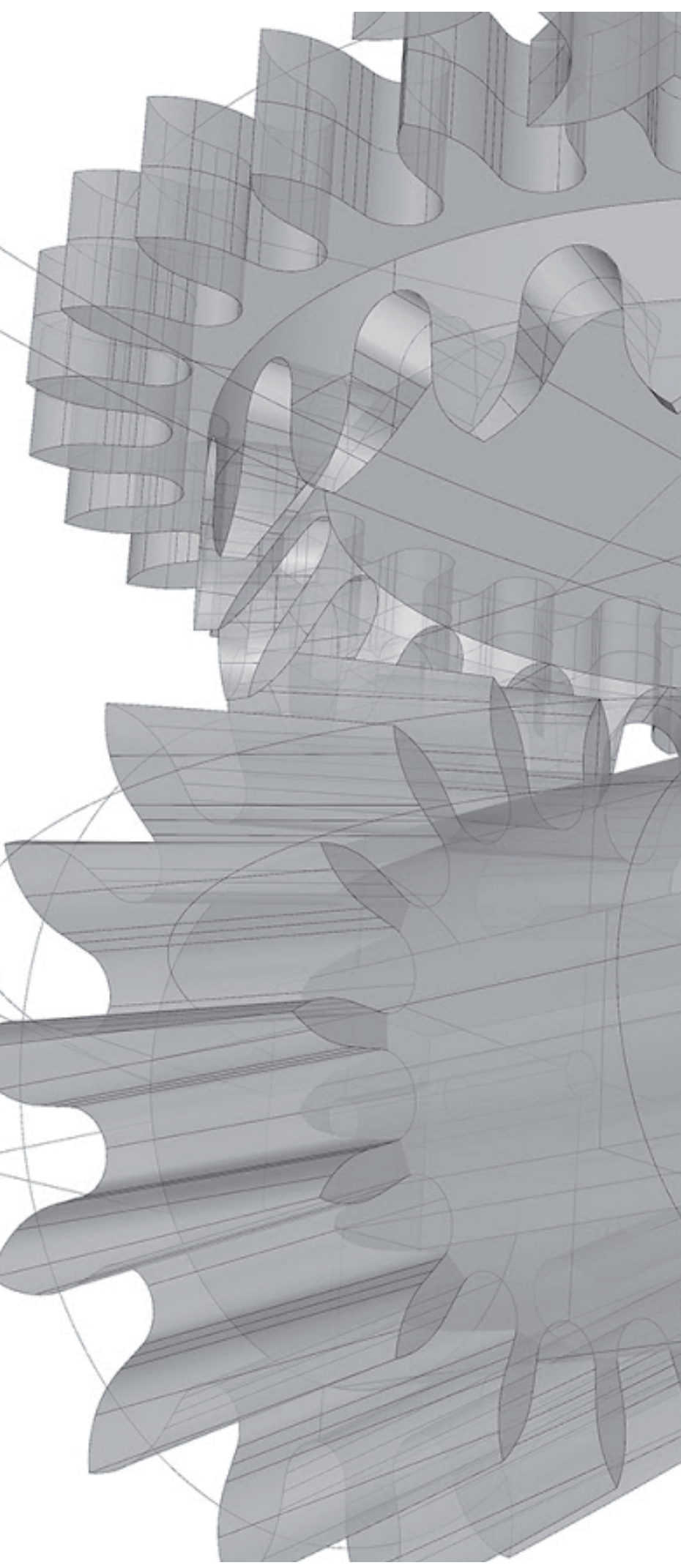




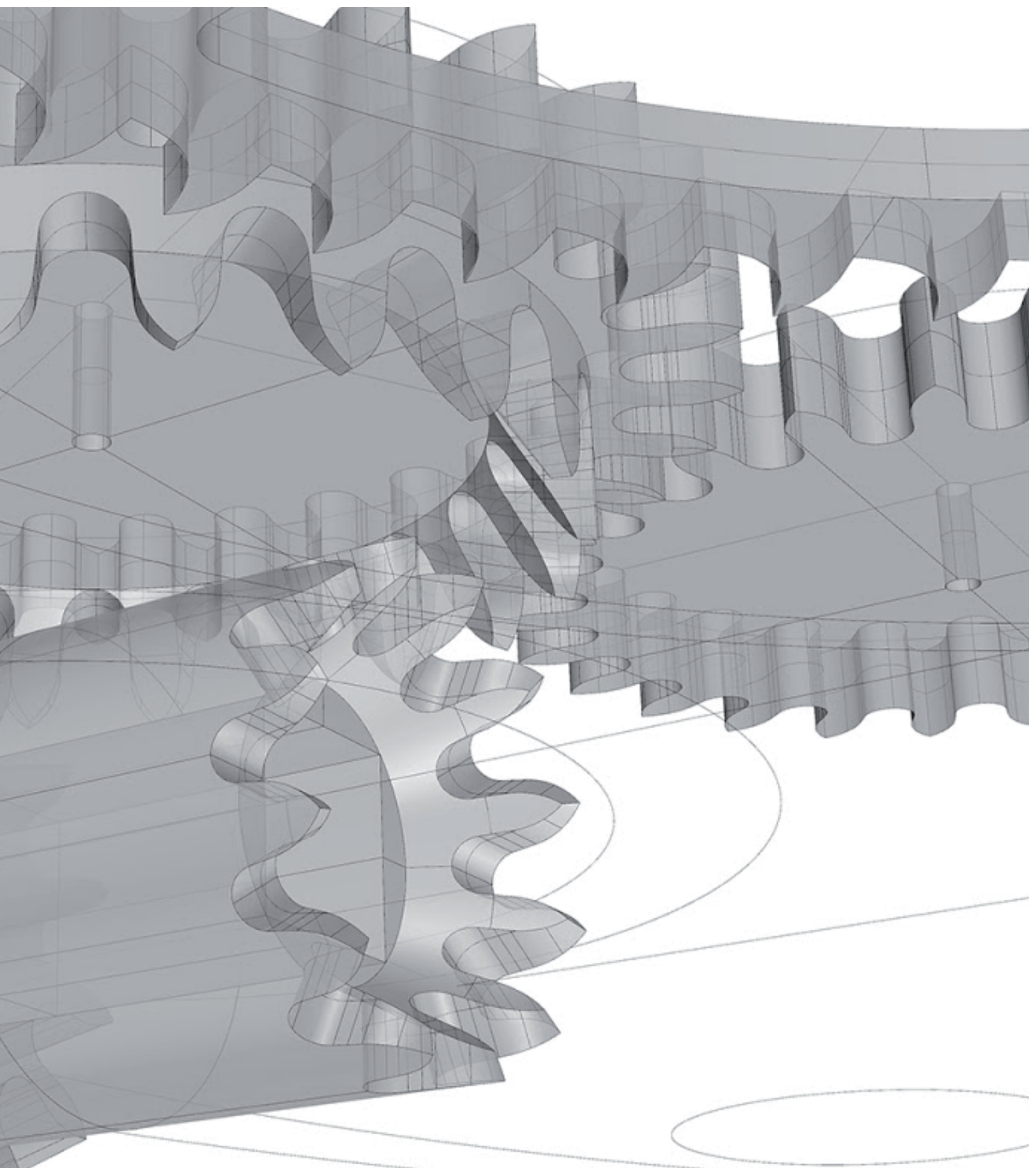

